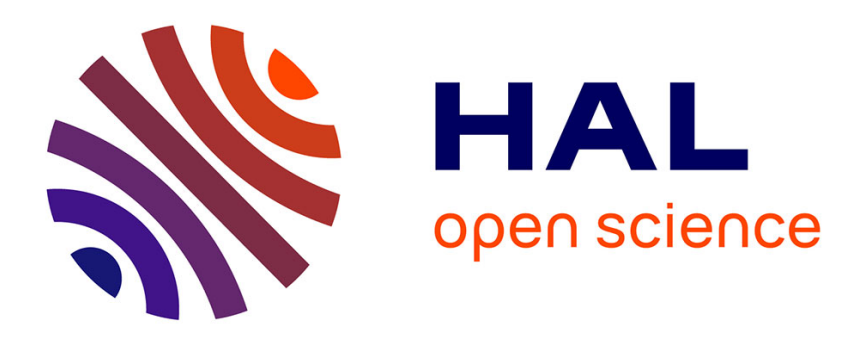

\title{
Différenciation, croissance et développement du tissu adipeux
}

\author{
J. Robelin, L. Casteilla
}

\section{To cite this version:}

J. Robelin, L. Casteilla. Différenciation, croissance et développement du tissu adipeux. Productions Animales, 1990, 3 (4), pp.243-252. hal-00895908

\section{HAL Id: hal-00895908 https://hal.science/hal-00895908}

Submitted on 1 Jan 1990

HAL is a multi-disciplinary open access archive for the deposit and dissemination of scientific research documents, whether they are published or not. The documents may come from teaching and research institutions in France or abroad, or from public or private research centers.
L'archive ouverte pluridisciplinaire HAL, est destinée au dépôt et à la diffusion de documents scientifiques de niveau recherche, publiés ou non, émanant des établissements d'enseignement et de recherche français ou étrangers, des laboratoires publics ou privés. 
INRA Prod. Anim., 1990, 3 (4), $243-252$

\section{J. ROBELIN et L. CASTEIILLA*}

INRA Laboratoire Croissance et Métabolisme des Herbivores

Theix, 63122 Saint-Genès-Champanelle

${ }^{\star}$ Adresse actuelle : CNRS,

12, rue Jules-Hetzel - 92190 Meudon

\section{Différenciation, croissance et développement du tissu adipeux}

\section{Résumé}

Le tissu adipeux est le principal organe de stockage d'énergie permettant d'assurer un équilibre entre les besoins et les apports chez de nombreux animaux. En dehors de ce rôle métabolique, il a un intérêt particulier chez les animaux producteurs de viande, car il détermine en partie la valeur commerciale de la carcasse et la qualité de la viande.

Le tissu adipeux est constitué de cellules appelées adipocytes, capables de synthétiser des acides gras, de les estérifier en triglycérides, et ultérieurement d'hydrolyser ces lipides pour mettre les acides gras à la disposition des autres tissus.

Son développement se déroule en trois étapes successives : prolifération cellulaire, différenciation et enfin grossissement des adipocytes. L'ontogenèse du tissu se fait à partir de cellules précurseurs (adipoblastes) qui se multiplient. Sous l'influence de gènes de détermination, ces cellules s'engagent dans le processus de différenciation et acquièrent en plusieurs étapes les caractéristiques fonctionnelles de l'adipocyte.

La mise en place des différents dépôts adipeux chez les bovins a lieu durant la vie foetale. Pendant cette période et durant le début de la vie postnatale, la prolifération des cellules adipeuses est très active. Ensuite, l'hypertrophie devient le facteur prépondérant de la croissance du tissu adipeux.

Les dépôts adipeux représentent environ $5 \%$ du poids du corps d'un veau nouveau-né. Ce pourcentage reste stable durant les 3 premiers mois de la vie postnatale, puis s'accroît de plus en plus rapidement et atteint environ $25 \%$ chez l'adulte.

Le développement des tissus adipeux ainsi que la répartition des dépôts dans les différents sites anatomiques sont variables selon le génotype des animaux et selon le niveau des apports alimentaires. Cependant, les connaissances sur la régulation de la différenciation et de la croissance du tissu adipeux ne sont pas encore suffisantes pour réaliser totalement la maîtrise de l'adiposité.
Le lissu adipeux joue le rôle d'un réservoir d'énergie, destiné à assurer l'équilibre instantané entre les besoins de l'animal (entretien, thermogenèse, locomotion, croissance, lactation..) et les apports alimentaires. Les conséquences pratiques de ce rôle sont nombreuses. C'est le tissu qui permet au ruminant en croissance ou en lactation de stocker des réserves pendant les périodes de surplus alimentaire, et de les utiliser durant les périodes de déficit; ces périodes respectives sont variables selon les climats considérés, et surtout selon l'intervention de l'homme dans l'approvisionnement de l'animal. Le tissu adipeux joue aussi un rôle important dans la détermination de la «qualité " des animaux de boucherie, et cela à deux niveaux. La qualité d'une carcasse à l'abattage est en partie appréciée sur la base de son état d'engraissement. L'échelle de valeurs peut d'ailleurs être variable selon les habitudes des régions et des pays. Enfin, une partie des qualités organoleptiques de la viande, et notamment la flaveur, semble étroitement dépendante des lipides intramusculaires, qui se développent en même temps (mais plus lentement) que les autres sites adipeux.

Cet article fait une synthèse des connaissances sur la mise en place et le développement des tissus adipeux. Après un bref rappel sur la structure du tissu adipeux et le rôle métaboli- 
que de l'adipocyte, nous présentons les principales étapes de la différenciation adipocytaire, puis les grandes lois de la croissance cellulaire et tissulaire des différents dépôts adipeux, et enfin les principaux facteurs de variation de leur importance chez les bovins.

\section{1 / Structure du tissu adipeux et métabolisme de la cellule adipeuse}

\section{1 / Structure du tissu adipeux}

Le tissu adipeux est constitué de cellules spécialisées, les cellules adipeuses, ou adipocytes, enfermées dans un treillis de fibres conjonctives. Ce tissu qui est en communication étroite avec les autres tissus et organes, est irrigué par un réseau dense de vaisseaux sanguins. $\mathrm{Au}$ niveau macroscopique, le tissu adipeux se développe préférentiellement le long des gros vaisseaux, ce qui est bien visible au niveau du tissu adipeux omental, localisé autour des préestomacs des ruminants.

La cellule adipeuse est une cellule de forme ovoïde, qui mesure une dizaine de microns de diamètre lorsqu'elle commence à stocker des lipides dans ses vacuoles, et plus de 150 microns lorsqu'elle est très hypertrophiée. Dans ces conditions, la cellule présente alors une énorme vacuole lipidique centrale, avec un novau rejeté sur un bord, dans un cytoplasme qui semble peu abondant en valeur relative (figure 1).

Figure 1. Description schématique de la cellule adipeuse.

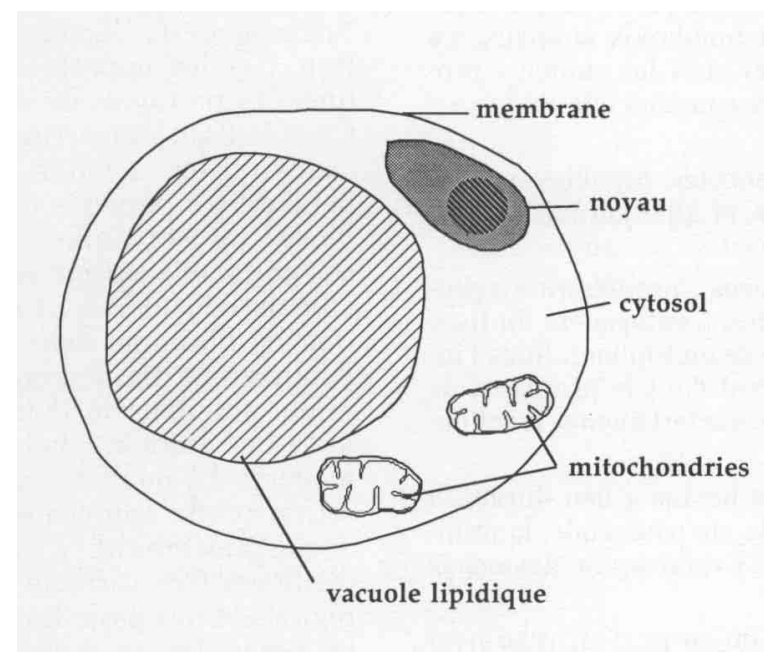

\section{2 / Rôle métabolique de l'adipocyte}

Les deux rôles principaux de la cellule adipeuse sont le stockage sous forme de lipides de l'énergie en excès, et la restitution de cette énergie en période de bilan négatif. Ces rôles sont résumés sur la figure 2.
Figure 2. Schéma de la lipogenèse et de la lipoIyse dans l'adipocyte.

Abréviations: TG: triglycérides; $A G L$ : acides gras libres; FAS: fatty acid synthétase; LPL: lipoprotéine lipase; VLDL: very low density lipoprotéin.

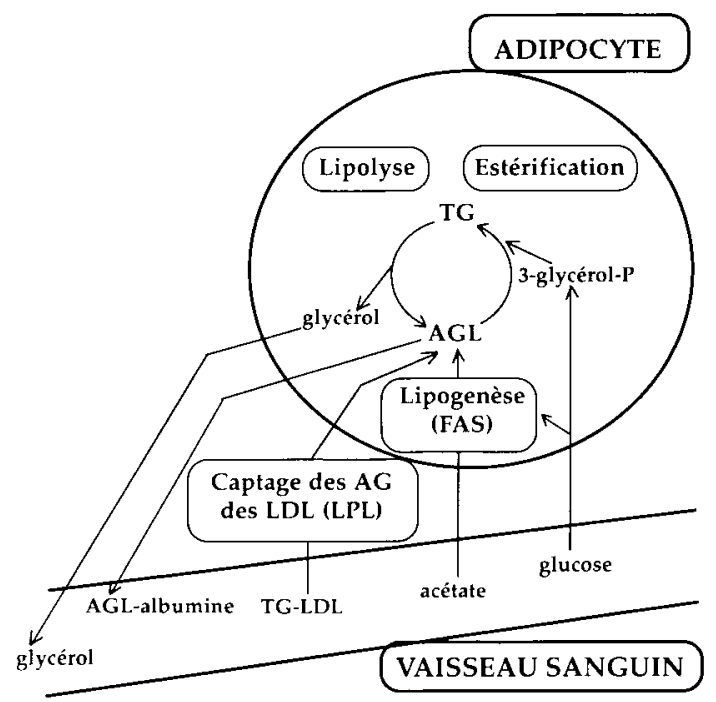

\section{a / Stockage de l'énergie excédentaire}

Les principales formes d'énergie circulantes sont les triglycérides et les phospholipides transportés dans les lipoprotéines, les acides gras non estérifiés et enfin les acides gras volatils. Lorsque l'animal est en bilan énergétique positif, le métabolisme de l'adipocyte est orienté vers la synthèse et le stockage des lipides, le message étant transmis par le relais de l'insuline notamment.

Le stockage comporte deux étapes successives: accroissement des acides gras libres dans l'adipocyte, puis estérification de ces acides gras en triglycérides.

Approvisionnement en acides gras. Il existe deux sources d'acides gras pour l'adipocyte, soit l'hydrolyse des lipides circulants grâce à la lipoprotéine-lipase, soit la synthèse de novo à partir des acides gras volatils absorbés (acétate notamment chez les ruminants). Chez les monogastriques, les acides gras sont synthétisés surtout à partir du glucose.

Estérification des acides gras. Cette réaction nécessite un apport de glucose pour la synthèse du radical glycérol des triglycérides.

\section{$b$ / Restitution de l'énergie}

Lorsque les conditions nutritionnelles ne suffisent pas à couvrir les besoins de l'animal, l'équilibre hormonal est modifié (diminution du taux d'insuline, accroissement de la sensibilité aux catécholamines), le flux d'énergie est inversé. Il y a hydrolyse des triglycérides en acides gras et glycérol grâce à la lipase hormono-sensible. Les produits de la lipolyse passent alors dans la circulation sanguine où le taux d'acides gras non estérifiées (AGNE) s'ac- 
croît ; ces précurseurs énergétiques sont ainsi mis à la disposition des tissus utilisateurs (muscles, cœur, foie...).

On trouvera plus de détails sur le métabolisme du tissu adipeux et sa régulation dans les revues de Bauman (1976), de Fain et GarciaSainz (1983), de Lafontan (1986) et de Chilliard (1987).

\section{2 / Ontogenèse et différenciation de la cellule adipeuse}

\section{1 / Etapes du développement cellulaire du tissu adipeux}

La formation du tissu adipeux débute par une phase de prolifération ou hyperplasie à partir de cellules précurseurs. La prolifération cellulaire ne peut intervenir que sur des cellules indifférenciées ou en cours de différenciation.

Après avoir acquis les caractéristiques qui leur sont propres, et en particulier l'équipement enzymatique nécessaire à la lipogenèse, ces cellules sont devenues des adipocytes. Elles sont alors capables de stocker des lipides et peuvent donc accroître leur volume (hypertrophie).

Chez des rongeurs soumis à un jeûne alimentaire sévère, la masse adipeuse ne diminue que par hypotrophie alors que le nombre d'adipocytes ne varie pas. Les observations effectuées chez des bovins vont dans le même sens. Il est donc important de caractériser les facteurs in vivo qui contrôlent la prolifération cellulaire, ainsi que ceux qui induisent les différentes étapes conduisant à l'adipocyte. A partir de la caractérisation de ces facteurs et de leur importance relative, on peut envisager d'agir de manière spécifique sur le développement de la masse adipeuse.

En résumé, il est possible d'agir sur le nombre ou sur la taille des cellules pour contrôler la croissance du tissu adipeux. La modification de la taille des adipocytes grâce à une restriction alimentaire est réversible, alors que l'on peut penser que le nombre de cellules qui peuvent devenir des adipocytes est fixé de manière quasi définitive dès le stade foetal, et ne peut pas être modifié ultérieurement.

\section{2 / La différenciation adipocytaire}

\section{a / Principes généraux de la différenciation cellulaire}

La différenciation cellulaire correspond pour une cellule à l'acquisition au cours du temps d'un phénotype déterminé (phénotype adipeux ou musculaire par exemple). Les cellules adipeuses et musculaires dérivent de cellules embryonnaires identiques à l'origine. Ces cellules originelles acquièrent de nombreuses protéines spécifiques au type cellulaire (protéines indispensables à la synthèse des lipides dans l'adipocyte, protéines nécessaires à la contraction musculaire pour les cellules musculaires par exemple). L'acquisition de ces protéines a lieu en réponse à la stimulation du génome (matériel héréditaire de la cellule) par différents facteurs tels que les hormones.

L'ensemble de ce processus est particulièrement complexe à étudier chez l'animal puisque ce phénomène intervient très précocement au cours du développement embryonnaire et foetal. Par ailleurs, la description précise des mécanismes et étapes à parcourir est difficile à caractériser in vivo car le type cellulaire à l'intérieur d'un tissu est hétérogène (cellules vasculaires, adipeuses, conjonctives) et soumis à un environnement cellulaire et tissulaire très complexe. C'est pourquoi la plupart des données concernant ce domaine de recherche ont été recueillies sur des systèmes de cellules en culture.

La différenciation correspond donc à la "transformation » d'une cellule sans caractéristiques particulières en une cellule spécialisée (adipeuse ou musculaire).

\section{b / Caractéristiques de l'adipocyte mature}

C'est une cellule capable de stocker des lipides sous forme de triglycérides et de les libérer à la demande de l'organisme (période de jeûne, de stress, d'excercice prolongé, de lactation). Outre les protéines indispensables au bon fonctionnement de toutes les cellules, ceci nécessite la présence d'au moins trois groupes de protéines plus ou moins spécifiques à l'adipocyte :

- les protéines qui permettent la captation, la synthèse et le stockage des lipides (lipoprotéine lipase, acide gras synthétase entre autres)

- les protéines qui permettent l'hydrolyse et la libération de ces lipides stockés (lipase hormono-sensible)

- les protéines nécessaires à la réception des signaux hormonaux émis par l'organisme (récepteurs à l'hormone de croissance, à l'insuline, aux catécholamines etc..) qui modulent les fonctions de l'adipocyte (stimulation ou inhibition de la lipolyse ou de la lipogénèse).

\section{c / Etapes de la différenciation adipocytaire}

Très tôt au cours du développement de l'embryon, l'expression de quelques gènes (certainement très peu nombreux et spécifiques à un phénotype) oriente une cellule à l'origine pluripotente vers le programme de différenciation adipocytaire : ce sont des gènes dits de détermination. Leur caractérisation est extrêmement récente et nous savons peu de choses sur eux. Les adipoblastes ainsi pré-déterminés et destinés à devenir des adipocytes, n'en ont cependant encore aucune caractéristique fonctionnelle, et sont capables de proliférer (figure 3). $\mathrm{Au}$ cours des multiplications cellulaires, certains adipoblastes acquièrent la capacité à s'engager dans la différenciation terminale (acquisition des enzymes de la lipogenèse, de la lipolyse, augmentation de la sensibilité à l'insuline) sous l'influence de certains facteurs et deviennent alors des préadipocytes.

Après un petit nombre de nouvelles multiplications cellulaires, ces cellules accumulent des lipides, deviennent ainsi des adipocytes, et sont à l'origine de foyers adipeux au sein du tissu. Cette dernière étape intervient progressivement 
Figure 3. Différenciation adipocytaire (d'après Ailhaud 1987).

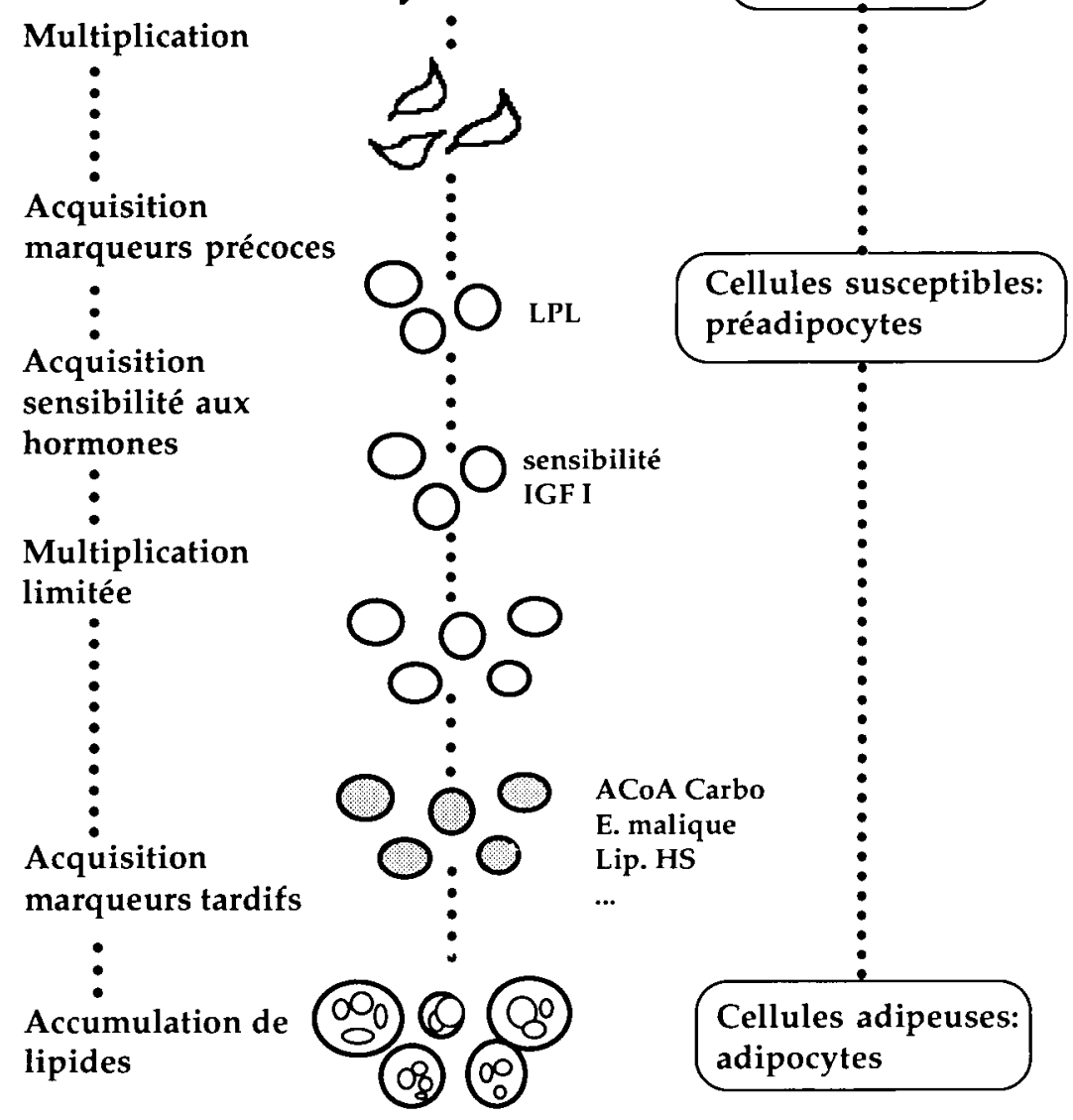

au cours du développement de l'animal et se poursuit même presque jusqu'au stade adulte où il subsiste encore un certain nombre de préadipocytes capables de se différencier et de participer à l'augmentation de la masse adipeuse.

La différenciation est donc un processus qui se déroule en plusieurs étapes dont la majorité a lieu très tôt chez l'embryon. Il n'existe pas à l'heure actuelle de mesure réelle du nombre de préadipocytes et à plus forte raison d'adipoblastes. Cependant, on sait qu'un certain nombre de préadipocytes, de moins en moins important avec l'âge, sont conservés tout le long de la vie de l'animal. Une des manières de limiter la croissance des tissus adipeux serait de freiner ou de supprimer totalement le recrutement de ces cellules.

\section{d / Régulation de la différenciation : signaux inducteurs, signaux modulateurs}

Afin que la différenciation se déroule normalement, il est indispensable que la cellule reçoive les signaux adéquats, le moment voulu et aux niveaux souhaités.

Une hormone est dite inductrice lorsque sa présence à un instant donné est indispensable à l'expression de certaines protéines, et donc d'un phénotype. C'est le cas de l'hormone de croissance $(\mathrm{GH})$ ainsi que de la Triodothyronine (T3) pour les adipocytes.

Une hormone est dite modulatrice quand le niveau d'expression des protéines, et donc du phénolype ultérieurement, est dépendant de la présence et de la concentration de celle-ci. C'est le cas de l'insuline et de la T3 dans certains cas et selon les espèces. Ces facteurs et leur influence sont variables selon les espèces et selon les sites adipeux. Les glucocorticoïdes seraient très importants chez les ovins et beaucoup moins chez les rongeurs. Les stérö̈des sexuels contrôleraient le niveau d'expression de certains types de récepteurs adrénergiques selon le site adipeux.

De nombreux autres facteurs non identifiés souvent secrétés par les adipocytes eux-mêmes (Insuline-like Growth Factor ou IGF...) et présents dans le sérum de veau foetal sont également indispensables à la différenciation.

On devrait pouvoir modifier le processus de différenciation et donc le nombre de cellules adipeuses en agissant sur le taux circulant ou local (à la périphérie de la cellule) de ces facteurs. En fait, les mécanismes mis en jeu sont probablement assez complexes, car chez un rat hypothyroïdien, le déficit en T3 et T4 s'accompagne d'une diminution transitoire du nombre d'adipocytes et d'une hypertrophie des cellules (régulation compensatrice de l'organisme?).

Ainsi, la différenciation cellulaire est contrôlée par de multiples facteurs. Ce n'est que la combinaison correcte de ceux-ci qui permet le bon déroulement du programme. La différenciation adipocytaire n'est pas sous l'influence d'un facteur adipogénique simple; il est donc difficile de mettre en oeuvre une maîtrise raisonnée et ciblée du développement de la masse adipeuse. Nous n'avons présenté ici qu'un résumé de la différenciation adipocytaire; on trouvera de plus amples délails dans les travaux de G. Ailhaud et collaborateurs, cités dans la bibliographie.

\section{3 / Croissance et développement du tissu adipeux des bovins}

Le lissu adipeux est probablement le tissu le plus variable et le plus «maléable». Les valeurs que nous indiquons dans le texte qui suit, sont données à titre d'illustration globale et font donc totalement abstraction de cette variabilité. Nous évoquerons dans un chapitre ultérieur cette variabilité selon le génotype ou le niveau alimentaire.

On envisagera tout d'abord la croissance cellulaire du tissu adipeux, puis développement à un niveau plus global.

\section{1 / Croissance cellulaire du tissu adipeux des bovins}

La mise en place des différents dépôts adipeux a lieu durant la vie foetale chez les bovins. Le tissu périrénal est visible dès le deuxième tiers de la vie foetale, les autres 
dépôts internes et les dépôts intermusculaires apparaissent vers le 6ème mois tandis que les dépôts sous-cutanés ne sont décelables que 1 ou 2 mois avant la naissance. Jusqu'à la naissance, les différents tissus adipeux présentent les caractéristiques du tissu adipeux brun. Ils renferment une protéine spécifique, appelée protéine découplante, dont la caractéristique est de permettre la production de chaleur à partir de l'oxydation des lipides de réserve. C'est par cette voie que le nouveau-né assure sa thermogenèse. Cette caractéristique disparaît chez le veau après quelques semaines de vie postnatale.

On distingue deux étapes dans le développement des tissus, la phase de multiplication cellulaire ou hyperplasie, et la phase d'accroissement de la dimension des cellules ou hypertrophie. Ces deux phases sont séparées par la différenciation qui met fin à la prolifération cellulaire. Cette distinction est abusive dans la mesure où l'on trouve simultanément dans un même tissu des cellules au niveau de ces deux étapes. Cependant cette simplification rend plus claire la description de la croissance du tissu.

\section{a / Accroissement du nombre de cellules (hyperplasie)}

La mesure du nombre réel de cellules adipeuses d'un dépôt est quasiment impossible. On apprécie le nombre total d'adipocytes d'un dépôt grâce au rapport entre le poids des lipides du dépôt (mesuré après dissection complète) et le poids d'une cellule adipeuse déterminé à partir du volume moyen des adipocytes. Dans la pratique on doit fixer la limite inférieure de taille des adipocytes pris en compte dans le calcul du volume moyen. Dans la suite de cet exposé, le nombre d'adipocytes correspond en fait au nombre de cellules de diamètre supérieur à 15 microns.

Le nombre d'adipocytes passe de 19 milliards vers la fin de la vie foetale à 124 milliards environ chez le bovin adulte (figure 4). La principale phase d'accroissement du nombre de cellules adipeuses est le début de la période postnatale, entre la naissance et le poids de $100 \mathrm{~kg}$. Soulignons qu'il s'agit d'une hyperplasie apparente, et plus exactement d'un accroissement du nombre d'adipocytes dont le diamètre est supérieur à 15 microns. Il est probable que la période d'hyperplasie réelle, c'est-à-dire de prolifération cellulaire est antérieure.

\section{b / Accroissement de la taille des adipocytes}

Le tissu adipeux renferme une population d'adipocytes dont le diamètre est très variable. Par exemple, chez un bovin atteignant $30 \%$ de son poids adulte, le diamètre des adipocytes varie de 25 à 100 microns, dans une population dont la répartition en fréquence est voisine d'une courbe de Gauss. Au fur et à mesure que l'animal stocke des lipides, la courbe de répartition se déplace dans la direction des diamètres plus élevés (figure 5). Pour des raisons de facilité, on caractérise la taille des adipocytes par le diamètre ou le volume moyen de cette population. Le diamètre moyen des adipocytes à la fin de la vie foetale est voisin de 40 microns (figure
Figure 4. Evolution du nombre et du diamètre des adipocytes chez les bovins au cours du développement (d'après Robelin 1985).
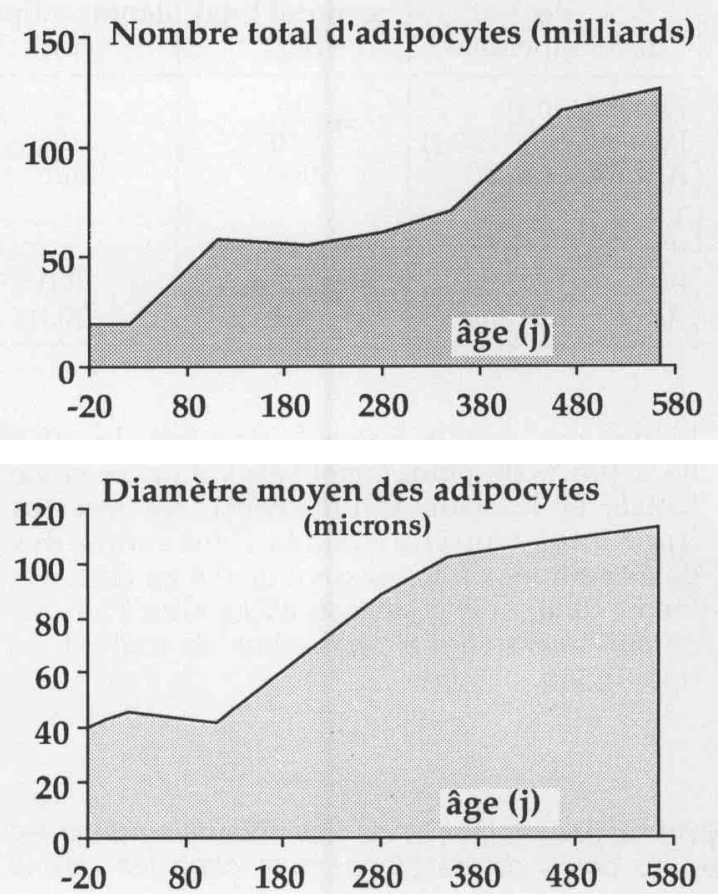

Figure 5. Répartition des adipocytes selon leur diamètre à deux stades du développement, 30 et $50 \%$ du poids adulte, chez les bovins (d'après Robelin 1981)

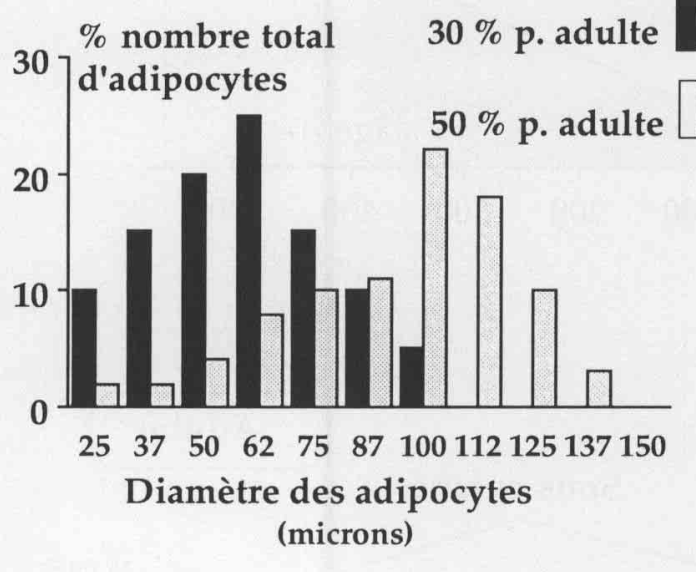

4). Il atteint une valeur de l'ordre de 120 microns chez le bovin adulte. Ainsi, le volume des cellules adipeuses s'accroît d'un facteur 30 environ, alors que le nombre de cellules ne s'accroît que d'un facteur 6 dans le même temps.

\section{c / Accroissement de la teneur en lipides du tissu adipeux}

La vacuole lipidique des adipocytes de petite taille est relativement peu importante par rapport au reste de la cellule. Au fur et à mesure que la cellule stocke des lipides, cette vacuole prend une importance relative grandissante. A un niveau plus global, la teneur moyenne en
La croissance tissulaire résulte de l'accroissement du nombre de ses cellules : hyperplasie et de leur taille : hypertrophie. 
Taktem . Croissance des tissus adipeux des bovins du stade fotal au stade adulte. (Robelin 1986, et résultats non publiés).

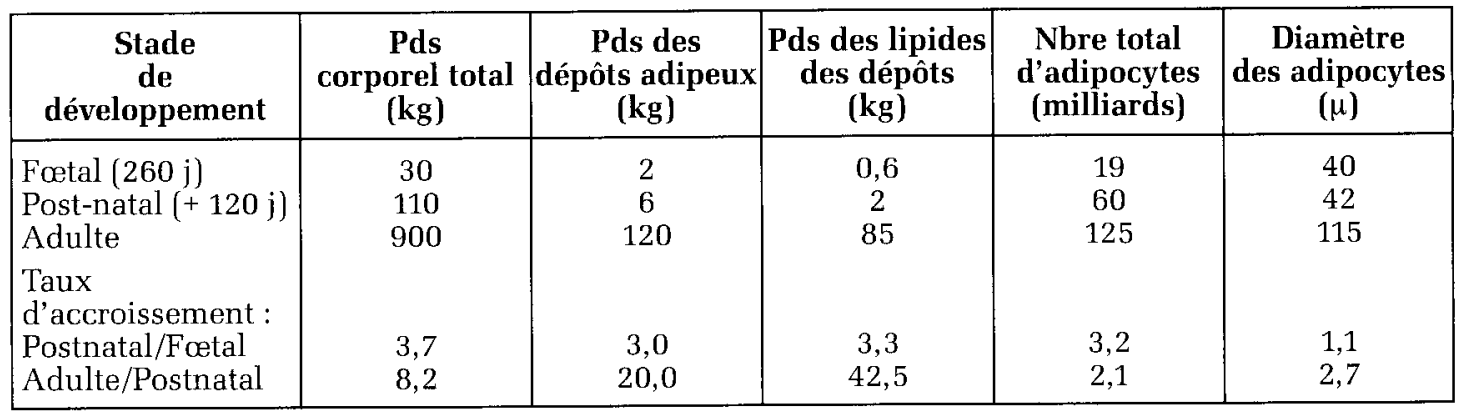

lipides des dépôts adipeux s'accroît de 30 à $80 \%$ (en \% du poids frais) entre la fin de la vie foetale et le stade adulte. Ainsi, les réserves lipidiques contenues dans l'ensemble des dépôts adipeux s'accroissent de 0,6 kg chez des foetus de 8 mois à environ $85 \mathrm{~kg}$ chez l'adulte, ce qui correspond à un facteur de multiplication de 140.

Evolution des dépôts adipeux totaux et de leur répartition dans les différents sites anatomiques au cours du développement chez les bovins (d'après Robelin 1985).
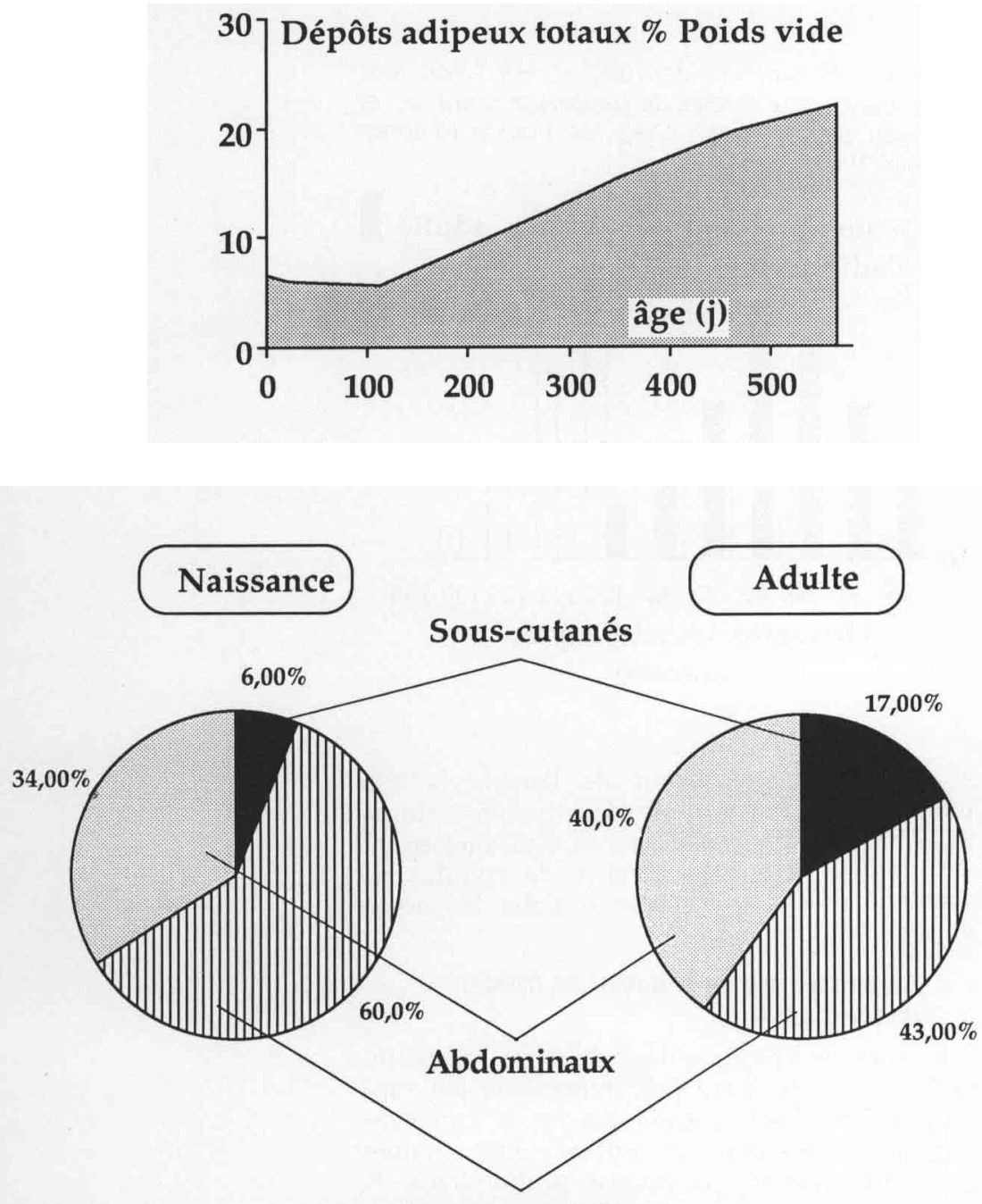

Intermusculaires

\section{d / Variabilité selon le site anatomique des dépôts adipeux}

Les valeurs que nous venons d'indiquer correspondent à l'ensemble des dépôts adipeux. Cependant, on observe des différences importantes dans le développement cellulaire selon les dépôts.

Ce sont les dépôts abdominaux (omentaux, périrénaux, mésentériques) qui renferment les adipocytes les plus gros (110 microns chez un bovin à $50 \%$ du poids adulte), et les dépôts sous-cutanés les plus petits ( 80 microns). Corrélativement, la teneur en lipides est également plus élevée dans les dépôts internes $(80 \%)$ que dans les dépôts externes (60\%).

En résumé (tableau 1), le poids des lipides contenus dans les dépôts adipeux s'accroît d'un facteur 140 environ entre la fin de la vie foetale et le stade adulte. Cet accroissement est dû essentiellement au remplissage de la vacuole lipidique des adipocytes et à l'accroissement de leur volume (x 30). Il est du à un moindre degré à l'augmentation du nombre d'adipocytes (x 6). Cette hyperplasie apparente est surtout active au début de la vie post-natale.

La conséquence la plus importante de cette part prépondérante de l'hypertrophie est que l'état d'engraissement est étroitement relié au diamètre des cellules adipeuses. Nous avons utilisé cette liaison pour mettre au point une méthode d'estimation in vivo de l'état d'engraissement des bovins, basée sur la mesure du diamètre des adipocytes d'un échantillon de dépôt sous-cutané prélevé par biopsie (Robelin et Agabriel 1986).

\section{2 / Croissance globale des dépôts adipeux des bovins}

Cet aspect de la croissance des tissus adipeux a fait l'objet d'un nombre considérable de travaux depuis ceux de l'Ecole de Hammond à Cambridge vers les années 50 (Callow 1950). Les objectifs de ces travaux étaient d'établir les lois de la croissance de ces tissus, afin de mieux maîtriser leur importance dans les carcasses des animaux à l'abattage. Le Laboratoire de la Production de Viande de l'INRA de Theix a apporté une contribution importante dans ce domaine.

On évoquera successivement l'évolution moyenne des dépôts au cours du développement, puis les principaux facteurs de variation 
de l'état d'engraissement à un stade de développement donné (race, sexe, niveau alimentaire...). Nous indiquons dans la bibliographie quelques articles les plus récents, qui synthétisent l'information abondante sur ce sujet.

\section{a / Evolution des dépôts adipeux au cours du développement}

On considérera dans ce paragraphe la croissance d'animaux continuellement alimentés à un niveau qui permette presque d'extérioriser totalement le potentiel de croissance, c'est à dire sans ralentissement du croit lié à une restriction alimentaire, et en corollaire sans période de croissance compensatrice.

Evolution de l'ensemble des dépôts adipeux. Le poids des dépôts adipeux totaux s'accroît de $5 \%$ du poids vide (sans contenu digestif) chez le nouveau né, à $25 \%$ environ chez le bovin mâle adulte de race Pie-noire (figure 6). Cependant, le croît des tissus adipeux est assez lent après la naissance dans les conditions normales d'élevage. Le pourcentage de dépôts dans le poids vide reste presque constant jusqu'à l'âge de 3-4 mois. A partir de cet âge, on assiste à un accroissement rapide du dépôt de lipides. Le coefficient d'allométrie des tissus adipeux passe alors de la valeur 1 à une valeur voisine de 2 chez les animaux au stade $70 \%$ du poids adulte. Ainsi, à partir du poids de $450 \mathrm{~kg}$, le croît journalier de dépôts adipeux dépasse même celui des muscles chez les animaux des races à forte adiposité telle que la Pie-noire.

La distinction de deux phases (croissance et engraissement) au cours du développement est surtout justifiée dans le cas (assez fréquent dans la pratique) où les animaux sont conduits avec un rythme de croissance modéré dans le jeune âge et reçoivent un régime beaucoup plus libéral durant une courte période avant l'abattage. Dans le cas d'animaux à croissance continue et rapide, la distinction de ces deux phases devient arbitraire: l'accroissement du dépôt de lipides est un phénomène continu, qui commence dès l'âge de 3 mois, et en tout cas bien avant ce que l'on appelle traditionnellement la phase d'engraissement.

Différences selon le site anatomique des dépôts adipeux. Nous avons déjà évoqué ces différences au niveau de la croissance cellulaire des dépôts. On peut comparer les différents dépôts selon deux critères indépendants, qui concernent l'importance respective de ces différents dépôts d'une part, et leur croissance relative par rapport à celle de l'ensemble de la masse adipeuse d'autre part.

Chez l'animal au stade $50 \%$ du poids adulte, les tissus adipeux intermusculaires (situés entre les muscles) représentent environ $55 \%$ de la masse adipeuse. On trouve ensuite par ordre décroissant, les dépôts sous-cutanés (15\%), omentaux $(10 \%)$, périrénaux $(7 \%)$ et mésentériques ( $6 \%$ ), puis d'autres dépôts de moindre importance.

Les tissus adipeux abdominaux et sous-cutanés ont une croissance relative très rapide. Leur proportion dans la masse adipeuse totale s'accroît entre la naissance et l'état adulte, de 7 à
$13 \%$ pour les tissus omentaux, de 4 à $9 \%$ pour les dépôts périrénaux et de 6 à $17 \%$ pour les dépôts sous-cutanés. En revanche, la proportion de dépôts intermusculaires décroît durant la même période de 60 à $43 \%$.

Nous n'avons pas évoqué jusqu'à présent les adipocytes situés entre les fibres musculaires, et qui constituent ce que l'on appelle le persillé de la viande. Ils ne forment pas un tissu comme les autres cellules adipeuses, et ne sont pas séparables à la dissection. On peut en apprécier l'importance grâce à la mesure de la teneur en lipides du tissu musculaire (lipides intramusculaires). Cette teneur s'accroît au cours du développment de 1,5 à $6 \%$ du poids frais de muscle, tandis que la teneur en lipides du corps entier s'accroît de 5 à $30 \%$ (figure 7).

La croissance différentielle des dépôts a des conséquences pratiques importantes. Tout d'abord, l'appréciation du gras de couverture est un bon reflet de l'état d'engraissement global, puisque la croissance relative des dépôts sous-cutanés est plus rapide que celle de la masse adipeuse. Par ailleurs, l'obtention d'une quantité suffisante de gras intramusculaire (persillé) est tributaire d'un état d'engraissement assez élevé.

Figure 7. Evolution des lipides corporels totaux et des lipides intramusculaires au cours du développement chez les bovins (d'après Robelin 1985).
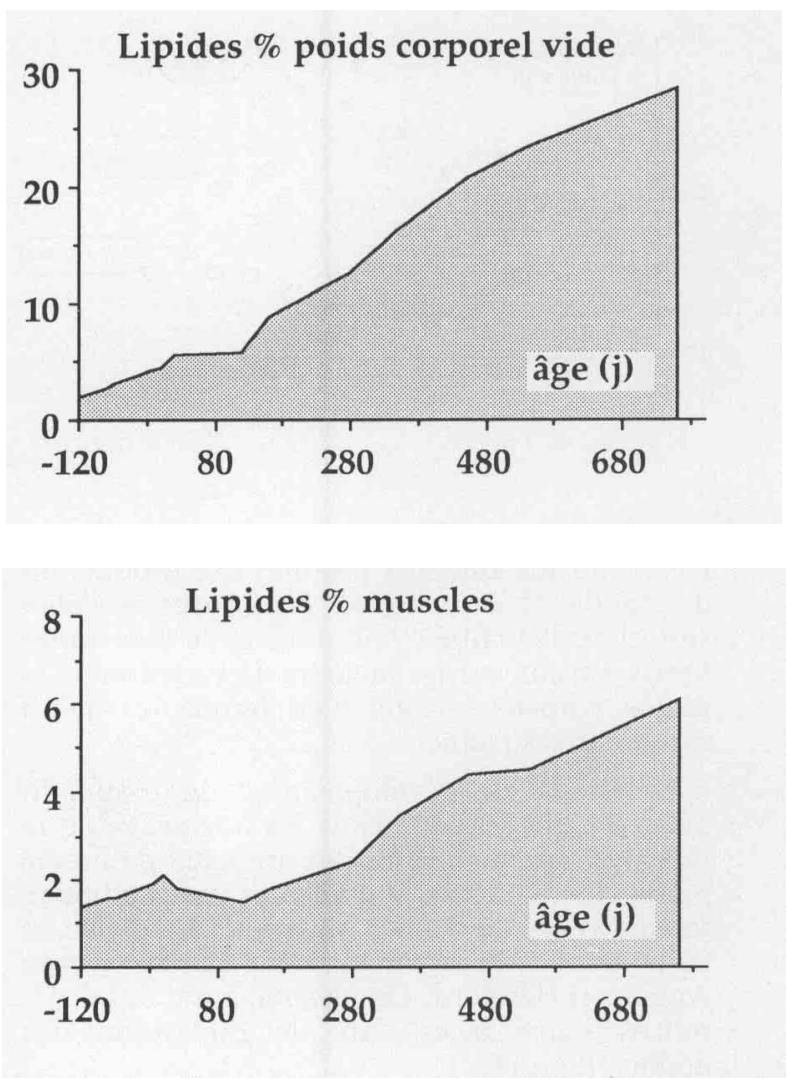

\section{b / Principaux facteurs de variation de l'état d'engraissement}

L'importance du tissu adipeux est beaucoup plus variable que celle des autres tissus. La recherche de la maîtrise de la croissance de ce

\section{Dans des conditions normales d'alimentation, le poids du tissu adipeux s'accroît régulièrement dès 3 mois et jusqu'à l'âge adulte.}


Figure 8. Variations de la répartition des tissus adipeux selon le génotype des bovins (analyse en composantes principales sur des résultats bibliographiques).

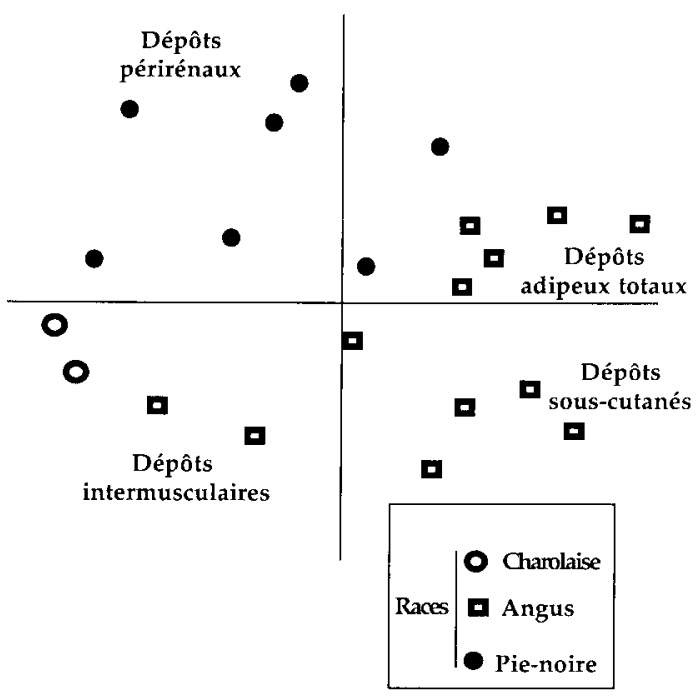

Figure 9. Variation de l'état d'adiposité des bovins selon le niveau d'alimentation. Interaction avec le génotype (schéma d'après une compilation de résultats bibliographiques).

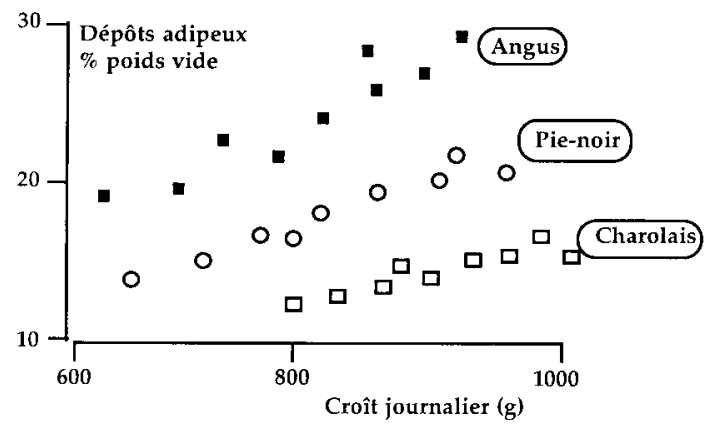

tissu chez les animaux destinés à la production de viande, et la recherche des causes de l'obésité chez l'Homme, ont motivé de très nombreux travaux sur les facteurs de variation de la masse adipeuse. Nous n'en ferons ici qu'un résumé très succinct.

La vitesse de développement du tissu adipeux est très variable chez les bovins, selon la race des animaux. Chez des animaux de même poids vide ( $450 \mathrm{~kg}$ ), le poids des tissus adipeux totaux varie de $72 \mathrm{~kg}$ en race Charolaise, à $90 \mathrm{~kg}$ en race Pie-noire et même $125 \mathrm{~kg}$ en race Angus ou Hereford. On observe aussi des différences entre races dans la répartition des dépôts (figure 8).

Les différences selon le sexe sont également bien connues. Les femelles renferment 30 à $60 \%$ de plus de tissus adipeux que les mâles entiers de même race au même stade de développement, tandis que les animaux mâles castrés occupent une position intermédiaire.
Enfin, il est possible de maîtriser la croissance des tissus adipeux grâce au niveau des apports alimentaires, et cela quelle que soit la période de croissance considérée. Ainsi, une restriction alimentaire pendant la période d'alimentation lactée de 0 à 3 mois chez des veaux Pie-noirs provoque une réduction du poids des lipides corporels au sevrage de près de $7 \mathrm{~kg}$, et une réduction de la taille des adipocytes. Une restriction alimentaire durant la période de croissance et d'engraissement produit également une réduction du poids des tissus adipeux à l'abattage, d'autant plus marquée que la réduction des apports est plus sévère et que la vitesse de croissance est plus faible (figure 9). Par ailleurs, cette réduction est plus importante chez les animaux des races à forte adiposité (Pie-noire), que chez ceux des races à viande plus maigres (Charolaise, Limousine). Cependant, cette réduction de l'adiposité n'est pas définitive, et peut être compensée totalement si les animaux sont à nouveau alimentés de façon libérale.

En résumé, l'éleveur dispose donc en théorie d'un certain nombre de moyens pour maîtriser l'adiposité des animaux, auxquels il faut ajouter les « anabolisants » qui ont souvent un effet sur le développement des tissus adipeux.

\section{Conclusion}

Le tissu adipeux, comme la plupart des autres tissus se met en place durant la vie foetale, et se développe ensuite au cours de la vie post-natale essentiellement par hypertrophie.

C'est un tissu dont la fonction physiologique de stockage de réserves d'énergie pour une utilisation ultérieure n'a qu'assez peu d'intérêt dans la plupart des systèmes de production de viande en pays tempérés. Son seul intérêt chez les animaux de boucherie est lié à l'appréciation commerciale des carcasses, et surtout la qualité de la viande (lipides intramusculaires).

Dans l'état actuel de l'utilisation des carcasses, l'objectif de l'éleveur est de produire un animal dont l'état d'engraissement est compris dans le créneau admis par le marché. Il faut donc définir des systèmes de production de viande, c'est-à-dire des rythmes de croissance et des régimes adaptés aux différentes catégories d'animaux (races, types sexuels) selon leur aptitude à déposer des lipides.

On peut envisager de maîtriser la masse adipeuse selon trois approches:

- contrôle des substrats nécessaires à la cellule adipeuse pour se développer

- contrôle des facteurs hormonaux qui régulent la lipogenèse et l'accroissement de la taille de la cellule

- contrôle du nombre de cellules adipeuses shez l'adulte.

La première de ces approches est le contrôle nutritionnel : réalisé et réalisable actuellement (en fin d'engraissement par exemple).

La deuxième est l'utilisation d'outils pharma- 
cologiques en pleine expansion ( $\beta$-agonistes, stéroïdes) dans la mesure où ils sont acceptés par la législation. Leur effet est pleïotropique, peu spécifique et pas toujours bien contrôlé ni exempt d'effets secondaires (activation cardiaque, modification de la qualité de la viande par les $\beta$-agonistes). Cependant, on peut penser que des substances plus spécifiques devraient apparaître prochainement (par exemple: agonistes ou antagonistes plus spécifiques à un type de récepteur très important dans la cellule adipeuse).

La troisième approche doit être envisagée mais nous connaissons encore peu de choses sur les moyens précis d'y parvenir. En effet, la différenciation est un dialogue structuré dans le temps entre le matériel héréditaire de la cellule et son environnement cellulaire et tissulaire. Dans ce contexte, un objectif pour la recherche sur la production de viande pourrait être de trouver un moyen de réduire le potentiel de développement de la population adipocytaire dans les différents dépôts, et au contraire de favoriser le développement des adipocytes entre les fibres musculaires. Cependant, il s'agit d'un objectif à long terme car on ne connaît pas encore avec précision les mécanismes qui déterminent le nombre de cellules précurseurs et donc le nombre des futurs adipocytes.

\section{Références bibliographiques}

Cette liste de références bibliographiques non exhaustive indique seulement des articles que l'on peut consulter pour une information plus précise sur les différents aspects traités. Ces références sont regroupées par thème afin d'en faciliter la recherche.

Métabolisme des lipides et sa régulation

BAUMAN D.E., 1976. Intermediary metabolism of adipose tissue. Fed. Proc., 35, 2308-2313

CHILLIARD Y., 1987. Variations quantitatives et métabolisme des lipides dans les tissus adipeux et le foie au cours du cycle gestation-lactation. Reprod. Nutr. Develop., 27, 327-398.

FAIN J.N., GARCLA-SAINZ J.A., 1983. Adrenergic regulation of adipocyte metabolism. J. Lip. Res., 24, 945-966.

LAFONTAN M., 1986. Physiologie et pharmacologie de la mobilisation des lipides : aspects actuels et futurs. Cah. Nutr. Diét., 21, 19-46.

\section{Différenciation adipocytaire et sa régulation}

AILHAUD G., 1987. Multiplication et différenciation des cellules adipeuses en culture. Médecine et Science, 3, 380-386.

AILHAUD G., ABBAD M., AMRI E., BARBARAS R., CERMOLACCE C., CZERUCKA D., DOGLIO A., FOREST C., GAILLARD D., GRIMALDI P., NEGREL R., VANNIER C., 1984. La différenciation adipocytaire en culture. Cah. Nutr. Diét., 19, 205-211.

AILHAUD G., AMRI E., BARBARAS R., CATALIOTO R.M., CZERUCKA D., DANI C., DESLEX S., DOGLIO A., FOREST C., GAILLARD D., GRIMALDI P., NEGREL R., VANNIER C., 1985. Différenciation des préadipocytes en adipocytes. Ann. Endocrin., 7, 1-7.

CASTEILLA L., FOREST C., ROBELIN J., RICQUIER D., LOMBET A., AILHAUD G., 1987. Characterization of mitochondrial uncoupling protein in bovine fetus and newborn calf. Am. J. Physiol., 252 (Endocrinol. Metab. 15), E627-E636.
CASTEILLA L., CHAMPIGNY O., BOUILLAUD F., ROBELIN J., RICQUIER D., 1989. Sequential changes in the expression of mitochondrial protein mRNA during the development of brown adipose tissue in bovine and ovine species. Biochem. J., 257, 665-671.

\section{Développement cellulaire du tissu adipeux}

KIRLAND J., GURR M.I., 1979. Adipose tissue cellularity $:$ a review. 2 . The relationship between cellularity and obesity. Int. J. Obesity, 3, 15-55.

ROBELIN J., 1981. Cellularity of bovine adipose tissues: developmental changes from 15 to 65 percent mature weight. J. Lip. Res., 22, 452-457.

ROBELIN J., 1985. Cellularité des différents dépôts adipeux des bovins en croissance. Reprod. Nutr. Dévelop., 25, 211-214.

ROBELIN J., AGABRIEL J., 1986. Estimation de l'état d'engraissement des bovins vivants à partir de la taille des cellules adipeuses. Bull. Tech. CRZV-Theix, INRA, 66, 37-41.

Croissance et développement global des dépôts adipeux

BERANGER $C$, ROBELIN J., 1977. Influence du mode d'élevage, de la sélection et de l'alimentation sur l'état d'engraissement des bovins. Ann. Biol. Anim. Biochim. Biophys., 17, 905-921.

CALLOW E.H., 1950. Comparative studies of meat. III Rates of fattening in relation to the percentage of muscular and fatty tissue in the carcass. J. Agric. Sci., 39, 347358.

GEAY Y., ROBELIN J., 1979. Variation of meat production capacity in cattle due to genotype and level of feeding : genotype nutrition interaction. Livest. Prod. Sci, 6, 263276.

KEMPSTER A.J., 1980. Fat distribution and partitioning in the carcasses of cattle, sheep and pigs : a review. Meat Sci., 5, 83-98.

ROBELIN J., 1978. Répartition des dépôts adipeux chez les bovins selon l'état d'engraissement, le sexe et la race. Bull. Techn. CRZV-Theix, INRA, 34, 31-34.

ROBELIN J., 1986. Growth of adipose tissues in cattle ; Partitioning between depots, chemical composition and cellularity. A review. Livest. Prod. Sci., 14, 349-364.

ROBELIN J., CHILLIARD Y., 1989. Short-term and long term effects of early nutritionnal deprivation on adipose tissue growth and metabolism in calves. J. Dairy Sci., 72, $505-513$.

\section{Summary}

Differentiation growth and development of adipose tissues.

Adipose tissue is the main energy buffer between the input of nutrients and the physiological needs of many animals. In meat producing animals, they have also a role in the determination of carcass and meat quality. This article is concerned with adipose tissue structure, adipose cell differentiation and finally with adipose tissue growth and development in cattle.

Three major steps are considered in the cellular development of adipose tissue : proliferation of precursor cells (adipoblasts), differentiation of these cells into functional adipose cells (adipocytes) and lastly enlargment of the adipocytes. All these developmental steps are under hormonal control.

The ontogeny of the various adipose tissues of cattle takes place during foetal life. During this period, these tissues contain uncoupling protein, specific to brown adipose tissue. This protein, which has an important role in the regulation of thermogenesis in new-born animals, disappears after birth. Cell proliferation is more active during foetal life, whereas cell 
hypertrophy mainly takes place after birth.

Adipose tissue represents $5 \%$ of the body weight of new-born calves and this remains constant for 3 months. Afterwards, this percentage increases more and more rapidly reaching approximately $25 \%$ in mature cattle. Subcutaneous and abdominal fatty tissues have a faster growth rate than intermuscular fat.

Development of adipose tissues, and fat parti- tion between anatomical sites are variable according to the breed of animals. Increasing (or decreasing) the level of food intake increases (or decreases) selectively adipose tissue growth, but this effect is mainly mediated by adipose cell size. The regulation of adipose tissue growth by the monitoring of adipose cell number is not yet possible.

ROBELIN J., CASTEILLA., 1990. Différenciation, croissance et développement du tissu adipeux. INRA Prod. Anim., 3 (4), 243-252. 